REVUE DE L'INSTITUT

FRANÇAIS D'HISTOIRE

EN ALLEMAGNE

\section{Revue de l'IFHA}

Revue de l'Institut français d'histoire en Allemagne

$4 \mid 2012$

IFHA 4

\title{
La Fondation Bosch et son action dans le domaine des relations franco-allemandes
}

\section{Peter Theiner}

Traducteur : Jean-Louis Georget

\section{OpenEdition \\ Journals}

Édition électronique

URL : http://journals.openedition.org/ifha/424

DOI : 10.4000/ifha.424

ISSN : 2198-8943

Éditeur

IFRA - Institut franco-allemand (sciences historiques et sociales)

Édition imprimée

Date de publication : 30 septembre 2012

Pagination : 124-132

ISSN : 2190-0078

Référence électronique

Peter Theiner, "La Fondation Bosch et son action dans le domaine des relations franco-allemandes », Revue de l'IFHA [En ligne], 4 | 2012, mis en ligne le 14 février 2013, consulté le 02 mai 2019. URL:

http://journals.openedition.org/ifha/424 ; DOI : 10.4000/ifha.424

Ce document a été généré automatiquement le 2 mai 2019.

(C)IFHA 


\title{
La Fondation Bosch et son action dans le domaine des relations franco- allemandes
}

\author{
Peter Theiner \\ Traduction : Jean-Louis Georget
}

Cette année est célébré le cinquantenaire du discours de Charles de Gaulle, et l'année prochaine, ce sera le traité de l'Élysée dont on commémorera l'anniversaire. Au sein du tandem franco-allemand, nous préférons aujourd'hui nous souvenir de victoires historiques et nous ne regrettons plus les défaites. Nous commençons à nous faire du souci lorsque nous percevons chez notre voisin des évolutions qui nuisent à l'amitié, qui affaiblissent le couple franco-allemand en Europe et mettent en danger l'unité européenne. Il convient de le dire immédiatement : parmi tous les problèmes auxquels le présent nous confronte, c'est celui pour lequel le monde entier nous envie. L'amitié franco-allemande a depuis pris le caractère de paradigme pour des pays qui veulent réévaluer leurs relations avec des adversaires d'autrefois. Cela vaut par exemple pour les citoyens au Japon et en Corée du Sud tout comme pour les partisans d'un partenariat entre la Pologne et l'Ukraine. En cela, l'image du tandem est aussi prégnante que pertinente. On n'avance avec succès qu'à partir du moment où les deux partenaires jouent leur rôle et l'on chute ensemble lorsque la volonté et la force d'avancer s'épuisent. Même pour les pays qui ne cherchent pas directement pour eux-mêmes des incitations et des exemples pour réussir à se réconcilier et à établir une amitié durable, la relation franco-allemande a valeur de modèle. Observer et commenter la coopération francoallemande sont devenus depuis longtemps des thèmes incontournables des think tanks de politique étrangère aux États-Unis. Dans la communauté stratégique transatlantique, on sait très bien que la cohésion et la stabilité européennes ne peuvent être obtenues sans une entente préalable entre Paris et Berlin, de sorte que l'Occident reste tributaire de cette entente. 
Cette évidence du tandem franco-allemand, dont l'environnement international a pris conscience, contraste occasionnellement de manière tranchée avec une tradition de diagnostic de crise qui accompagne la relation franco-allemande depuis ses origines. Avant la parenthèse bien médiatisée d'une réconciliation tournée vers l'avenir, c'est d'abord une tradition de séparation intellectuelle qui a plutôt accompagné l'histoire riche en conflit des deux voisins rhénans, depuis l'époque de la Révolution française jusqu'au milieu du XXe siècle, lui donnant sa tonalité de fond.

C'est sous des auspices contraires que se dessine aujourd'hui le diagnostic de crise intellectuelle entre les deux protagonistes: il ne dramatise plus aujourd'hui à coups de formules rhétoriques l'identité nationale propre pour se démarquer de celle du voisin. Nous tombons plus prosaïquement sur des évidences qui pointent la fragilité du tandem. C'est ainsi que Pierre Nora, l'un des historiens français les plus en vue, a exprimé il y a peu dans la Frankfurter Allgemeine Zeitung ses inquiétudes quant à la décrépitude progressive de la coopération franco-allemande. Il accusait la faiblesse de l'apprentissage de la langue du voisin dans les deux pays, la distance des intellectuels, qui avaient moins à se dire qu'autrefois, les structures disparates des systèmes d'enseignement supérieur, et pour finir, une recrudescence générale de l'individualisme dans nos sociétés ainsi qu'une tendance à la renationalisation des cultures européennes - tout ceci en reconnaissant que la culture allemande lui était malheureusement relativement étrangère.

Certes, les temps sont révolus dans lesquels la langue du partenaire occupait une place particulièrement privilégiée dans chacun des cursus nationaux ; l'acquisition de la langue du voisin stagne dans les deux pays à un niveau dont l'appréciation peut varier en fonction du point de vue. En fait la langue de l'autre est soumise aujourd'hui en tant que langue étrangère apprise à l'école à d'autres conditions de concurrence que celles qui existaient encore dans les années 1980. Il n'est sans doute pas vraisemblable de penser que le choix des langues étrangères puisse être opéré en vertu d'un lien particulièrement étroit avec l'affection que l'on entretient pour l'autre pays, il répond bien plus pour les parents et les élèves à pléthore de motifs et d'intérêts qui n'ont que peu à voir avec l'intérêt que l'on porte au voisin.

Dans les universités allemandes également, la prééminence du français dans les études romanes ne va plus aujourd'hui de soi. En observant cette tendance, on ne doit pas voir de blâme outrancier pour les relations franco-allemandes, puisqu'il s'agit bien plus de phénomènes courants en matière de mutation culturelle, en tout cas pour ce qui concerne la France et l'Allemagne, d'événements de différenciation et de pluralisme accompagnant la mondialisation. On peut simplement voir par exemple dans l'avancée de l'espagnol, mais encore plus vraisemblablement dans celle de langues et de cultures exotiques comme le chinois ou le japonais, un enrichissement de nos cultures. Quoi qu'il en soit, cette évolution n'est en aucun cas le symptôme d'un éloignement franco-allemand, puisqu'il s'agit bien plutôt d'un changement qui suit les lois propres des systèmes de formation et les tendances économiques qui les accompagnent.

Dans le domaine des échanges en sciences humaines et sociales, il n'y a pas plus de raison d'être pessimiste lorsqu'on y regarde de plus près. Au contraire, il existe depuis longtemps un réseau serré de partenariat entre les universités allemandes et françaises, qui a trouvé depuis l'année 2000 grâce à la création de l'Université franco-allemande une nouvelle structure commune très novatrice. Depuis, ce sont 130 formations binationales de premier cycle et plus de 40 cursus de master binationaux qui y ont trouvé leur alma mater. Nous pouvons parler aujourd'hui d'une véritable percée de la mobilité académique, 
car, jamais auparavant, autant d'étudiants n'avaient osé faire le saut dans une université du pays partenaire. L'Université franco-allemande est entièrement innovante en ce sens que les échanges conduisent à des doubles diplômes reconnus - et ce dans de nombreuses disciplines par-delà les sciences humaines et sociales qui ont toutes toujours été orientées vers l'international. De cette façon, l'UFA a produit un vivier tout à fait nouveau d'ingénieurs, de scientifiques, d'économistes et de juristes dont la biographie universitaire a été profondément imprégnée par l'amitié franco-allemande. Pour prendre en compte de la manière la plus objective et la plus réaliste le tandem, il est important de jeter un regard sur les réalités académiques en dehors des disciplines herméneutiques, dans lesquelles la réflexion et la recherche sur la base de coopérations franco-allemandes ont toujours été de mise - c'est-à-dire de jeter un regard sur des champs du savoir dans lesquels Allemands et Français apprennent, enseignent et recherchent aujourd'hui en collaborant avec une évidence toute naturelle.

Une observation de ce qui se passe dans l'espace public montre que l'amitié repose sur un réseau dense d'amitié grâce à environ 2400 jumelages entre les villes et de nombreux autres groupements associatifs au-delà des partenariats officiels. Certes, les rapprochements entre villes et groupements de la société civile n'ont pas tous la même vigueur, mais on pourrait faire la même remarque pour de nombreuses associations et communautés dans d'autres domaines de nos sociétés. Ils suivent des conjonctures, connaissent des périodes fastes de leur développement, avant que de nouveaux vecteurs plus jeunes ne les suppléent et fassent en sorte que la dynamique perdure.

De fait, le danger de renationalisation, sur lequel Pierre Nora attire notre attention, doit être minutieusement scruté. Il ne peut provenir aujourd'hui d'une idéologie de masse chargée de démagogie et d'hostilité : il s'agit bien plus, en y regardant de plus près, d'un risque lié à des divergences de priorités et d'objectifs, notamment dans les domaines de la politique budgétaire, financière et économique. Dans les années à venir, il nous revient de faire que les élites politiques en Allemagne et en France continuent de promouvoir avec soin la culture éprouvée du compromis et agissent en suivant les rails de la convergence. Contrairement au début des années 1980, lorsque les conceptions en matière de politique économique des gouvernements de Paris et Bonn divergeaient largement, sans toutefois que la substance de la coopération franco-allemande n'en subisse les dommages, nous avons à traiter aujourd'hui en Europe des problèmes économiques qui ne permettent pas de phases hasardeuses d'expérimentation économique ou bien de politiques prenant des détours égoïstes afin de satisfaire la simple prospérité nationale. Les deux plus grandes économies de la zone euro sont étroitement impliquées ne serait-ce que par la monnaie commune avec l'ensemble des autres nations, de sorte que tout pas en direction d'une renationalisation aurait des conséquences difficilement calculables - de la même manière qu'une extension exagérée de la solidarité européenne pourrait conduire sur un chemin au terme duquel les citoyens perdraient leur confiance dans le projet européen.

L'histoire de la réussite de l'amitié et de l'entente franco-allemandes a une paternité multiple. La conviction de Konrad Adenauer selon laquelle « il ne subsiste aucun doute que nous appartenions au monde ouest-européen en vertu de notre origine et de notre façon de penser " (20 septembre 1949 au Bundestag) est aujourd'hui reconnue dans notre pays par-delà les appartenances partisanes. À cette stratégie et aux valeurs qu'elle porte appartiennent un ancrage irréfutable dans les structures européennes, un renoncement à la souveraineté et une réconciliation basée sur une coopération avec la France comme noyau d'un continent toujours plus intégré. Dans le même temps, il est de plus en plus 
clair que la politique d'entente réciproque menée a aussi besoin d'un soubassement mental solide dans la société pour s'y rattacher et pour s'y appuyer. Cette dernière peut d'ailleurs occasionnellement servir de moteur.

Lorsqu'une histoire d'amitié entre les peuples est trop bien organisée et se fige, nous savons qu'elle conduit à une impasse. Ce sont nécessairement bien plus les personnes et les groupes qui inventent un autre avenir de leur propre initiative au moyen de leur pensée et leur action issue au-delà de l'horizon fixé. Ils laissent ainsi grandir de ce fait un capital social (Bourdieu). Dans l'entre-deux-guerres, nous trouvons de nombreuses traces d'une telle orientation vers le futur. Ce que nous appelons aujourd'hui société civile commença à émerger dans le domaine des relations internationales sous les motifs tacites suivants : la politique étrangère est trop importante pour la laisser exclusivement aux exécutifs nationaux. La recherche historique a dessiné entre-temps une image très différenciée des efforts des acteurs issus de la société civile dans le domaine de l'entente entre les peuples. "Faire entrer dans le jeu la société civile des deux nations»: les initiatives de réconciliation du XXe siècle ont suivi cette recommandation de la fondation Saint-Simon qui date de 1996. La "catastrophe originelle " de la Première Guerre mondiale a été essentielle pour les avocats de la coopération franco-allemande et la volonté de rechercher un nouveau départ, de résoudre les conflits selon des règles bien édictées et de contribuer à la détente par-delà toutes les divergences objectives d'opinion - et ce dans un climat public dans lequel les graves conséquences de la guerre semblaient pouvoir bloquer comme un mur infranchissable toute tentative de réconciliation.

L'entrepreneur et le fondateur Robert Bosch incarne les efforts pour parvenir à un équilibre franco-allemand de la manière la plus impressionnante. La base matérielle de son engagement citoyen a été l'ascension légendaire de son entreprise dont il avait fait dès avant la Première Guerre mondiale un Global Player. Des traces biographiques anciennes montrent que Robert Bosch était en outre persuadé que la vie active ne peut pas s'épuiser dans une action managériale. Il était tout aussi convaincu que le progrès et la prospérité ne peuvent pas être obtenus par la simple bonne volonté, mais doivent être cultivés dans une communauté libre où règne l'esprit de compétition que permet l'économie de marché. Dans des déclarations précoces du fondateur, le mot " adaptation » apparaît comme un terme clé, certainement pas au sens d'ancrage dans ce qui existe déjà, mais comme un devoir de s'atteler à de nouveaux défis. Il suivait cette maxime en faisant en sorte, lui qui était un chef d'entreprise couronné de succès, de faire participer les employés de ses usines de manière équitable à ses succès professionnels. Il affirmait de façon consensuelle qu'il devait sa richesse à ses équipes qu'il rémunérait au-delà de la moyenne et ne prétendait en aucun cas le contraire ! « Sois un être humain et prends soin de la dignité humaine !». C'était également un leitmotiv de son parcours personnel. Dans le domaine des efforts transfrontaliers pour arriver à se comprendre mutuellement, il en arriva à l'idée qu'ils ne devaient pas simplement être le pré carré des élites éclairées, mais que cette entente entre les peuples devait concerner les hommes dans les couches moyennes de la société.

Il prit pour ce faire le publiciste Paul Distelbarth sous contrat. Ce dernier commença à voyager à partir du printemps 1932 entre l'Allemagne et la France pour y tenir des discours tant il maîtrisait bien l'art oratoire et pour faire passer auprès du public l'un de ces messages essentiels: les «différences entre les Allemands et Français sont plus imaginaires que réelles ». C'était une simplification laconique, mais voulue. Elle aurait pu émaner de la plume de Robert Bosch lui-même, qui possédait la capacité légendaire à 
saisir de manière plutôt pragmatique la réalité de l'individu vivant et de la résumer dans son essence en quelques phrases. Robert Bosch saisissait les ressorts des fondements économiques et politiques réels des relations franco-allemandes avec le regard simple d'un industriel et d'un bourgeois éclairé. Il prenait en particulier au sérieux les soucis et les intérêts français en termes de sécurité, comprenant la crainte des voisins d'outre-Rhin devant les incertitudes allemandes et devant le risque que la politique étrangère allemande puisse de nouveau quitter le sentier balisé de la politique de réconciliation menée par Aristide Briand et Gustave Stresemann pour choisir la voie d'une politique hégémonique se refusant à tout compromis.

Pour Robert Bosch, la réponse était la suivante : la diplomatie allemande devait aller au devant de la France pour atteindre ce qui était possible par des voies pacifiques en tendant la main et avec une conscience claire. La gravité de l'heure exigeait « une certaine mesure dans les exigences » et non pas une politique hasardeuse et attentiste au regard du danger du nationalisme exacerbé qui croissait de nouveau. C'est ainsi que Robert Bosch écrivait encore en octobre 1933 au ministre des Affaires étrangères, c'est-àdire peu de jours avant que l'Allemagne ne quitte la société des nations, sans pouvoir savoir que les jalons du régime hitlérien étaient depuis longtemps posés pour provoquer un conflit.

Le fait de vouloir à tout prix la réconciliation franco-allemande été inséré pour Robert Bosch dans une vision européenne qui voyait l'intégration de son pays dans des structures européennes qui étaient encore à déterminer. Il encourageait l'équilibre franco-allemand, mais également un mouvement paneuropéen et formulait à la charnière des années 1932 et 1933 l'espoir d'une «Europe unie et pacifique » en évoquant dans le même contexte «l'unification entre l'Allemagne et la France » comme « la tâche la plus urgente ».

Au début des années 70, c'est-à-dire dans le contexte historique du traité de l'Élysée, la fondation Robert Bosch s'est placée sur les traces du fondateur et a mis en place un axe franco-allemand, le premier dans le domaine de la réconciliation entre les peuples, qui a été suivi jusqu'à aujourd'hui par beaucoup d'autres axes thématiques liés à d'autres pays, notamment la Pologne, que la fondation Robert Bosch a reçu dans son ressort alors qu'on était encore en pleine Guerre froide.

On demande de temps à autre s'il est encore nécessaire aujourd'hui de faire des efforts particuliers pour renforcer la relation franco-allemande, que ce soit dans le domaine des rencontres pour jeunes, dans le cadre des séminaires communs pour journalistes, dans les programmes pour les traducteurs, dans les écoles d'été pour les cadres ou pour toutes les autres initiatives. Nous disions au début que le monde entier envie les Français et les Allemands pour avoir réussi leur réconciliation et leur rapprochement et fait de leur amitié et de leur bon voisinage une évidence. Beaucoup d'autres pays attendent surtout, pas seulement en Europe, que les deux voisins rhénans montrent une responsabilité politique commune en Europe et la conduisent avec une certaine modestie, une prise en compte de tous les intérêts et une certaine mesure. Le pouvoir politique ne peut prendre en compte cette responsabilité que s'il se sait soutenu par l'assentiment tacite des citoyens. C'est donc pour cette raison que nous avons besoin de rencontres, de liens humains, de projets communs au quotidien, et pas seulement pour les commémorations.

La fondation Bosch est donc l'une des plus grandes fondations entrepreneuriales d'Allemagne qui met au service du rapprochement et de la réconciliation entre les peuples les moyens dont elle dispose dans les domaines de la formation et de la santé au 
sens où l'entendait son fondateur Robert Bosch (1861-1942). La fondation existe dans sa forme actuelle depuis 1964. En 2011, 68 millions d'euros ont été octroyés pour des programmes et des projets communs et d'utilité publique.

La fondation se concentre pour ce qui concerne son axe franco-allemand et les relations qu'il implique sur les thèmes de l'engagement citoyen, de la culture et de la langue ainsi que sur l'encouragement aux médiateurs comme les journalistes et les cadres. Les moyens mis au service de ces encouragements sont utilisés par exemple pour :

- le concours d'idées franco-allemands «On y va - Auf geht's!» qui encourage les projets communs de groupes de la société civile française et allemande.

- «Deutschmobil » et « FranceMobil » qui permet à des diplômés de l'université de rapprocher de manière ludique la culture et la langue des jeunes et des enfants des deux pays voisins.

- le « dialogue d'avenir entre la France et Allemagne ", grâce auquel les cadres des deux pays peuvent discuter des défis actuels dans les relations franco-allemandes et rédiger des recommandations pour l'action politique.

- le programme «Gemeinsam mehr Chancen - Avancer ensemble » pour lequel on facilite les conditions de mobilité touchant la formation professionnelle au plan régional.

Depuis la mise en place de l'axe franco-allemand en 1973, ce sont 42,1 million d'euros qui ont été mis à disposition des initiatives franco-allemandes. La fondation DVA, qui n'est pas une fondation autonome à l'intérieur de la fondation Robert Bosch, mais une «filiale» s'engage à soutenir et promouvoir les relations franco-allemandes dans le domaine de la culture, de la littérature du théâtre. Depuis 1997, elle a mis 4,3 millions d'euros à la disposition des programmes engagés. Voir le site : www.bosch-stiftung.de

\section{AUTEURS}

\section{PETER THEINER}

Peter Theiner est responsable du département Völkerverständigung Europe de l'Ouest/Amérique à la Robert-Bosch Stiftung et directeur de la DVA-Stiftung, filiale de la Fondation Bosch. 